Corresponding author: jaster@ partners.org

(c) 2015 Kluk et al. This article is distributed under the terms of the Creative Commons Attribution-NonCommercial License, which permits reuse and redistribution, except for commercial purposes, provided that the original author and source are credited.

Ontology terms: dysplastic granulopoiesis, leukemia

Published by Cold Spring Harbor Laboratory Press

doi: $10.1101 /$ mcs.a000307

\section{Myeloid neoplasm demonstrating a STAT5B-RARA rearrangement and genetic alterations associated with all-trans retinoic acid resistance identified by a custom next-generation sequencing assay}

\author{
Michael J. Kluk, ${ }^{1}$ Ryan P. Abo, ${ }^{2}$ Ronald D. Brown, ${ }^{1}$ Frank C. Kuo, ${ }^{1}$ Paola Dal Cin, ${ }^{1}$ \\ Olga Pozdnyakova, ${ }^{1}$ Elizabeth A. Morgan, ${ }^{1}$ Neal I. Lindeman, ${ }^{1}$ Daniel \\ J. DeAngelo, ${ }^{2}$ and Jon C. Aster ${ }^{1}$ \\ ${ }^{1}$ Department of Pathology, Brigham and Women's Hospital, Boston, Massachusetts 02115, USA; ${ }^{2}$ Department \\ of Medical Oncology, Dana-Farber Cancer Institute, Boston, Massachusetts 02215, USA
}

\begin{abstract}
We describe the case of a patient presenting with several weeks of symptoms related to pancytopenia associated with a maturation arrest at the late promyelocyte/early myelocyte stage of granulocyte differentiation. A diagnosis of acute promyelocytic leukemia was considered, but the morphologic features were atypical for this entity and conventional tests for the presence of a PML-RARA fusion gene were negative. Additional analysis using a custom next-generation sequencing assay revealed a rearrangement producing a STAT5B-RARA fusion gene, which was confirmed by reverse transcription polymerase chain reaction (RT-PCR) and supplementary cytogenetic studies, allowing the diagnosis of a morphologically atypical form of acute promyelocytic leukemia to be made. Analysis of the sequencing data permitted characterization of both chromosomal breakpoints and revealed two additional alterations, a small deletion in RARA exon 9 and a RARA R276W substitution, that have been linked to resistance to all-trans retinoic acid. This case highlights how next-generation sequencing can augment currently standard testing to establish diagnoses in difficult cases, and in doing so help guide selection of therapy.
\end{abstract}

\section{INTRODUCTION}

Ongoing technologic advances such as next-generation sequencing (NGS) continue to exponentially increase DNA-sequencing capacity and improve sequencing accuracy, while at the same time reducing costs. As a result, NGS is playing an increasing role in the diagnosis and subclassification of cancer in the clinic. One diagnostic area that remains difficult is the workup of patients with unexplained cytopenias or cytoses, abnormalities that are commonly seen in clinical practice. Generally, in cases in which blasts are found in the peripheral blood or bone marrow in large numbers it is straightforward to establish a diagnosis of acute leukemia. However, many patients with abnormal blood counts present without increased blasts, and other conventional tests, such as karyotyping and flow cytometry, also may 
fail to reveal abnormalities. Over time, these patients may progress to an overt myeloid neoplasm such as a myelodysplastic syndrome or a myeloproliferative neoplasm, or may remit, presumably because the underlying cause is extrinsic to bone marrow progenitors. It is also recognized that the marrows of some patients without blasts nevertheless harbor clonal abnormalities that are usually associated with overt acute leukemia; by convention, such cases are treated like acute leukemia, even in the absence of typical morphologic findings.

Here, we report the case of a patient with pancytopenia whose workup revealed findings consistent with an unclassifiable myeloid neoplasm associated with a maturation arrest at the promyelocyte/myelocyte phase of granulocyte differentiation. Analysis of marrow DNA using a custom NGS assay revealed a STAT5B-RARA fusion gene, a rare molecular abnormality reported previously in fewer than 10 cases of acute promyelocytic leukemia (APL) (Arnould et al. 1999; Kusakabe et al. 2008; Swerdlow et al. 2008; Iwanaga et al. 2009; Chen et al. 2012; Strehl et al. 2013). The morphologic findings in our case were atypical for APL, suggesting that similar cases associated with STAT5B-RARA fusion genes may have gone unrecognized. In addition, STAT5B-RARA-associated APL is reported to be resistant to therapy with all-trans retinoic acid (ATRA), unlike some other molecular subtypes of APL, but the underlying mechanisms are unclear. By sequencing both breakpoints and the RARA coding sequence, we also identified two RARA sequence variants that have been linked to resistance to all-trans retinoic acid.

\section{RESULTS}

The patient is a 47-year-old man who presented with bruising, gingival bleeding, and fatigue over several weeks. He was found to have mild pancytopenia, hypofibrinogenemia, and a mildly increased prothrombin time/INR (international normalization ratio) (Table 1). His past medical history was remarkable only for hyperlipidemia and supraventricular tachycardia; he had no personal or family history of blood disorders or leukemia.

Review of the patient's peripheral blood smear revealed immature granulocytes, including myelocytes and rare promyelocytes (Fig. 1A). A bone marrow aspirate revealed an increased ratio of granulocyte-to-erythroid precursors and a predominance of myeloid forms at the promyelocyte/myelocyte stage, including very rare promyelocytes with single Auer rods (Fig. 1B), and the concurrent bone marrow biopsy (Fig. 1C) revealed a markedly hypercellular specimen ( $<5 \%$ adipose tissue) containing a predominant population of intermediate size mononuclear cells consistent with myelocytes and promyelocytes. Flow cytometry of aspirated marrow showed an expanded population of myeloid cells with surface expression of CD45(dim), CD13, CD33(variable expression), and CD117(dim, subset) that were negative for HLA-DR and CD34.

Based on the clinical presentation (bleeding, hypofibrinogenemia) and morphologic and flow cytometric findings, APL was suspected and the patient was immediately started on ATRA therapy $\left(45 \mathrm{mg} / \mathrm{m}^{2}\right)$ in divided daily doses. Cytogenetic analysis revealed the loss of chromosome $Y$ in a majority of cells $(45, X,-Y[15] / 46, X Y[5])$. A fluorescence in situ hybridization test for the presence of a PML-RARA rearrangement was negative using the PML/ RARA Dual Color, Dual Fusion Translocation probe (Abbott Molecular, Des Plaines, IL) (nuc ish $(P M L, R A R A) \times 2$ [100]). Reverse transcription polymerase chain reaction (RT-PCR) tests were also negative for $P M L-R A R A$ and $B C R-A B L 1$ fusion genes. In light of the negative tests for a PML-RARA fusion gene, ATRA was stopped after $2 \mathrm{~d}$.

Taken together, the constellation of the clinical and pathologic findings were difficult to assign to any single unifying diagnosis, although an unclassifiable myeloid neoplasm was favored; however, Auer rods (taken to be specific for myeloid neoplasms) were only discovered 
Table 1. Complete blood counts and coagulation studies at presentation

\begin{tabular}{lcr}
\hline & Result & \multicolumn{1}{c}{ Units } \\
\hline WBC & 2.1 & $10^{3}$ cells $/ \mu \mathrm{L}$ \\
Lymphocytes & 47 & $\%$ \\
Neutrophils & 30 & $\%$ \\
Monocytes & 18 & $\%$ \\
Metamyelocytes & 3 & $\%$ \\
Myelocytes & 1 & $\%$ \\
Bands & 1 & $\mathrm{~g} / \mathrm{dL}$ \\
Hemoglobin & 12.5 & $\mathrm{fL}$ \\
Hematocrit & 37 & $\mathrm{cells} / \mu \mathrm{L}$ \\
MCV & 83.3 & $\mathrm{mg} / \mathrm{dL}$ \\
Platelets & 135 & $\mathrm{Sec}$ \\
Fibrinogen & 128 & $\mathrm{Sec}$ \\
PT & 16.7 & \\
PTT & 30.8 & 1.4 \\
INR & 1.4 & \\
\hline
\end{tabular}

WBC, white blood cell; MCV, mean corpuscular volume; PT, prothrombin time; PTT, partial prothrombin time; INR, international normalization ratio.

in rare cells on later review of the case, and at the time of presentation a reactive process leading to a granulocyte maturation arrest (e.g., due to a medication or an environmental toxin) was also entertained. Because of the lack of a definitive diagnosis, the patient was initially managed with close clinical follow up over several weeks. His course was complicated by cytokine cascade activation (elevation in C-reactive protein, erythrocyte sedimentation rate, serum IL2 receptor, and ferritin), which was managed with dexamethasone (10 mg twice daily); fever (in the face of a negative infectious disease workup); and brachiocephalic vein

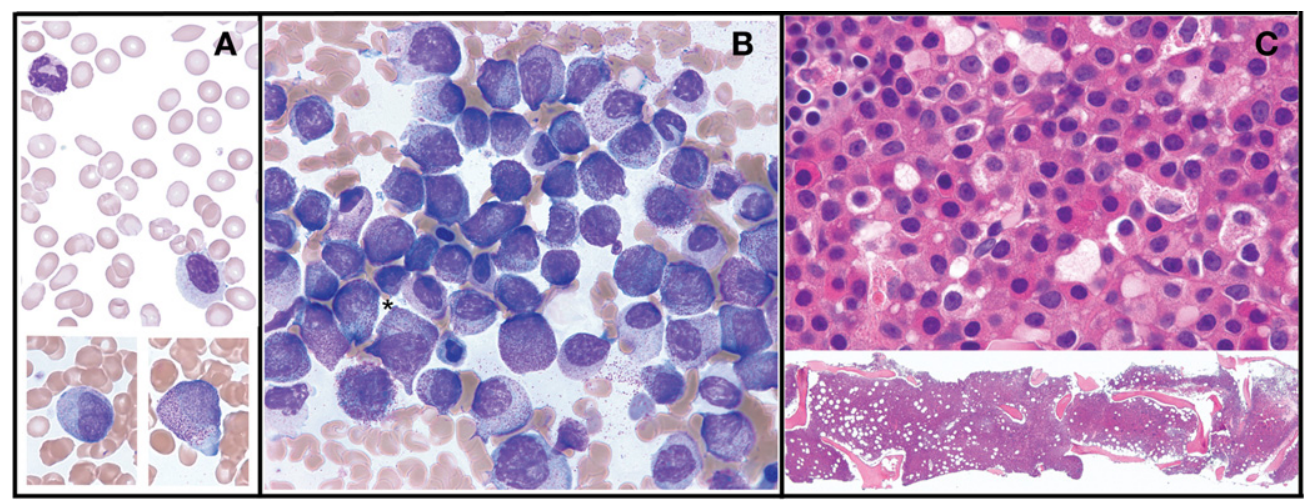

Figure 1. Peripheral blood and bone marrow findings. (A) Peripheral blood showing neutrophils, myelocytes, and rare forms resembling promyelocytes that have variable cytoplasmic granularity (bottom panel). (B) Bone marrow aspirate showing a predominance of myelocytes mixed with occasional promyelocytes, including rare forms with a single Auer rod (asterisk). (C) Bone marrow core biopsy showing marked overall hypercellularity (bottom panel) and a predominance of early and midstage myeloid forms. All images were taken with $100 \times$ oil objective except for $C$, bottom inset ( $2 \times$ objective); SPOT Insight Gigabit Digital Camera and SPOT Imaging Software 5.1. 
thrombosis (managed with low molecular weight heparin and rivaroxaban). While on dexamethasone, the patient's white blood cell count rose transiently due to increased numbers of circulating neutrophils, metamyelocytes, and myelocytes. A rheumatological workup revealed no evidence of collagen vascular disease or other abnormality. During this period, the patient's performance status improved.

In the interim, a somatic mutational profile of the patient's bone marrow sample at presentation was generated with a custom NGS gene-panel assay ("OncoPanel") (Wagle et al. 2012; Abo et al. 2015). Single-nucleotide variants, small insertions/deletions (indels) and copy number alterations were assessed in 300 genes that are recurrently mutated in various malignancies. Additionally, evaluation of the NGS data for structural alterations in 35 genes that are recurrently rearranged in cancer was performed using BreaKmer software (Abo et al. 2015), as well as by manual review. This additional analysis revealed a rearrangement/inversion involving STAT5B and RARA (Fig. 2; see Table 2 for contig sequences), which are both located on Chromosome 17. The presence of this rearrangement was subsequently confirmed by PCR of genomic DNA for breakpoint A and RT-PCR of cDNA followed by Sanger sequencing for breakpoint B (Fig. 3; see Table 3 for PCR primers and conditions) (Chen et al. 2012). Notably, sequencing of the breakpoint B RT-PCR product confirmed the presence of a STAT5B-RARA inversion (Fig. 3).

These findings were also consistent with the presence of a deletion of part of the inverted DNA at breakpoint A (Figs. 3 and 4) encompassing STAT5B exon 16 though the $3^{\prime}$ end of RARA exon 9 (encoding amino acids L409 to P462), with preservation of the

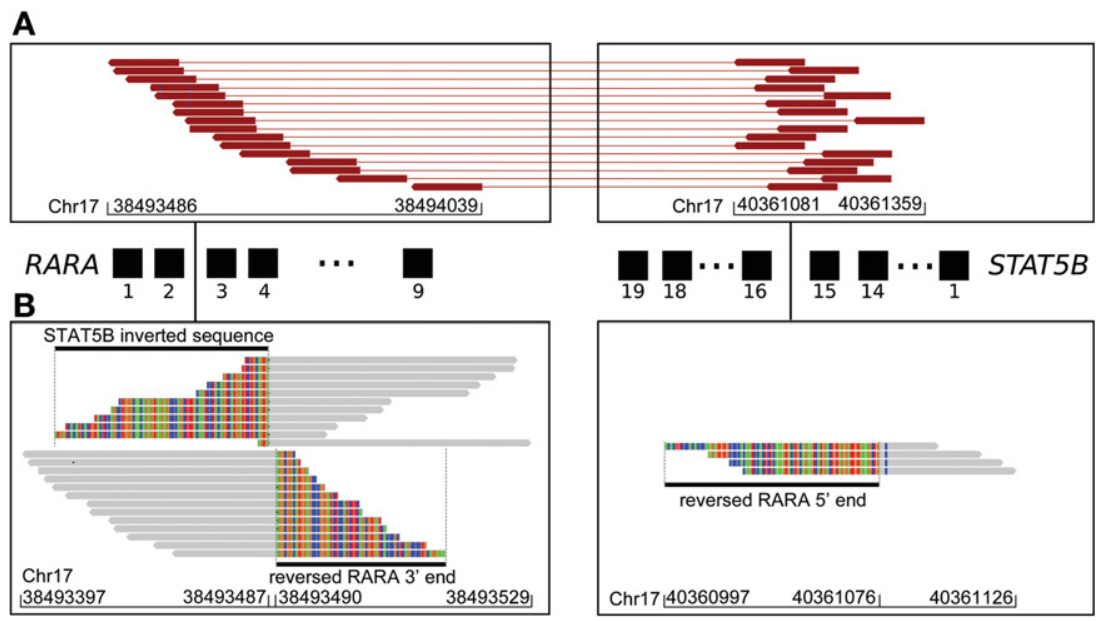

Figure 2. Identification of STAT5B-RARA fusion gene resulting from an inversion event by NGS. (A) Discordant STAT5B-RARA paired reads. Mapped paired-end sequenced reads (red rectangles) are shown in which one read mapped to RARA and their respective mate to STAT5B (black boxes indicate exons with their respective numbers); read pairs are shown with red lines between the reads. The discordance in the mapping distance between the read pairs ( 2 Mb), as well as their orientation (reverse-reverse), provides evidence for an inversion. Tapered ends of the mapped reads pointing left indicate the mapped orientations in the reverse direction. Genomic coordinates for the left and right boundaries of mapped reads are provided. (B) STAT5B-RARA and RARA-RARA fusion reads that span rearrangement breakpoints. Portions of these reads are mapped to the reference sequence (gray color) and an unrelated sequence (multicolored). ( $B$, Left panel, top cluster) reads spanning breakpoint $B$ (see Fig. 4) containing sequences mapping to RARA (gray) and to STAT5B (multicolored nucleotides, in reverse orientation); ( $B$, Left panel, bottom cluster) reads spanning RARA breakpoint $A$ (see Fig. 4) that mapping RARA exon 9 (gray) and to the 3' portion of RARA (multicolored nucleotides, in reverse orientation); (B, Right panel) additional reads spanning breakpoint B of Figure 4 mapping to STAT5B (gray) and 5' portions of RARA (multicolored nucleotides in reverse orientation). Images were generated with Integrative Genomics Viewer (IGV) software, version 2.3. 
Table 2. Genomic alterations identified by next-generation sequencing

\begin{tabular}{|c|c|c|c|c|c|c|c|c|}
\hline \multicolumn{9}{|c|}{ Single-nucleotide variants } \\
\hline Gene & Chr & HGVS DNA & $\begin{array}{l}\text { HGVS } \\
\text { protein }\end{array}$ & Variant type & $\begin{array}{l}\text { Variant allele } \\
\text { frequency }\end{array}$ & $\begin{array}{l}\text { Predicted } \\
\text { effect }\end{array}$ & $\begin{array}{l}\mathrm{dbSNP} \\
\mathrm{ID}\end{array}$ & Genotype \\
\hline RARA & 17 & c. $826 \mathrm{C}>\mathrm{T}$ & p.R276W & Substitution & $\begin{array}{l}13 \% \text { of } 61 \\
\text { reads }\end{array}$ & Pathogenic & N/A & Heterozygous \\
\hline \multicolumn{9}{|c|}{ Copy number alterations } \\
\hline \multicolumn{9}{|l|}{ None } \\
\hline \multicolumn{9}{|c|}{ Rearrangement analysis } \\
\hline Genes & Chr & HGVS DNA & & HGVS protein & Variant type & Predicted effect & $\begin{array}{l}\mathrm{dbVar} \\
\mathrm{ID}\end{array}$ & Genotype \\
\hline STAT5B-RARA & 17 & $\begin{array}{l}\text { t(17;17)(q21.2. } \\
\text { (hg19 Chr17: } \\
\text { g.40361080_ }\end{array}$ & $\begin{array}{l}21.2) \\
493462)\end{array}$ & N/A & Inversion & $\begin{array}{l}\text { STAT5B-RARA } \\
\text { Fusion }\end{array}$ & N/A & N/A \\
\hline \multicolumn{9}{|l|}{ Breakpoint A } \\
\hline \multicolumn{9}{|l|}{ Fusion Contig } \\
\hline \multicolumn{9}{|c|}{$\begin{array}{l}\text { GAGGACACAGTGCTGGGGGAGGTTAAAGTACTGCAGAATCATGACTGTAGGATTGTAAAGCTGGGCTGTTTTCCACCATCTACATAGGC } \\
\text { AGCTTAGGCGGCATGGAGCCCGGGATCTCCATCTTCAGCGTGATCACCGCTCAGCCCCTGCAGGAAA }\end{array}$} \\
\hline \multicolumn{9}{|c|}{ Intron 2, RARA, 17:38493397-38493490 } \\
\hline \multicolumn{9}{|c|}{$\begin{array}{l}\text { GAGGACACAGTGCTGGGGGAGGTTAAAGTACTGCAGAATCATGACTGTAGGATTGTAAAGCTGGGCTGTTTTCCACCATCTA } \\
\text { CATAGGCAGCTT }\end{array}$} \\
\hline \multicolumn{9}{|c|}{ Exon 9, RARA, 17:38512261-38512313 } \\
\hline \multicolumn{9}{|c|}{ AGGCGGCATGGAGCCCGGGATCTCCATCTTCAGCGTGATCACCGCTCAGCCC } \\
\hline \multicolumn{9}{|c|}{ Intron 8, RARA, 17:38512260-38512251 } \\
\hline \multicolumn{9}{|l|}{ CTGCAGGAAA } \\
\hline \multicolumn{9}{|l|}{ Breakpoint B } \\
\hline \multicolumn{9}{|l|}{ Fusion Contig } \\
\hline \multicolumn{9}{|c|}{$\begin{array}{l}\text { AAAGTACTGCAGAATCATGACTGTAGGATTGTAAAGCTGGGCTCTTACTCTGTTGCCCAGGCTGGAGTACAGTGATGTGATCATGACTC } \\
\text { Intron 2, RARA, 17: 38493421-38493462 }\end{array}$} \\
\hline \multicolumn{9}{|c|}{ AAAGTACTGCAGAATCATGACTGTAGGATTGTAAAGCTGGGC } \\
\hline GAGTCATGAT & CAT & TGTACTCCA & CCTGGGC & CAGAGTAAGA & & & & \\
\hline
\end{tabular}

The segments within the Breakpoint A fusion contig and within the Breakpoint B fusion contig are indicated by normal text, gray shading, black shading, or bold underlined text and correspond to the matching genomic coordinates provided for each segment.

5' portion of RARA exon 9 (encoding amino acids G391 to P408). Interestingly, the deletion involving exon 9 of RARA removes a portion of the coding sequence for the retinoic acid binding domain and overlaps with small deletions that have been associated with ATRA resistance (i.e., RARA deletions spanning E412 to L414) (Tomita et al. 2013). Additionally, NGS revealed a RARA R276W missense variant (Table 2) that also has been reported to be associated with ATRA resistance (Takayama et al. 2001; Tomita et al. 2013). Notably, both of these alterations were identified in a marrow sample obtained prior to ATRA treatment.

The molecular evidence of the STAT5B-RARA rearrangement prompted additional fluorescence in situ hybridization on the patient's original marrow sample using a RARA dual color, break apart probe (Abbott Molecular). This revealed an unbalanced rearrangement in 91\% of cells, which retained signals corresponding to the $5^{\prime}$ centromeric RARA probe and lost signals corresponding to the $3^{\prime}$ telomeric RARA probe, compatible with the expected deletion of 


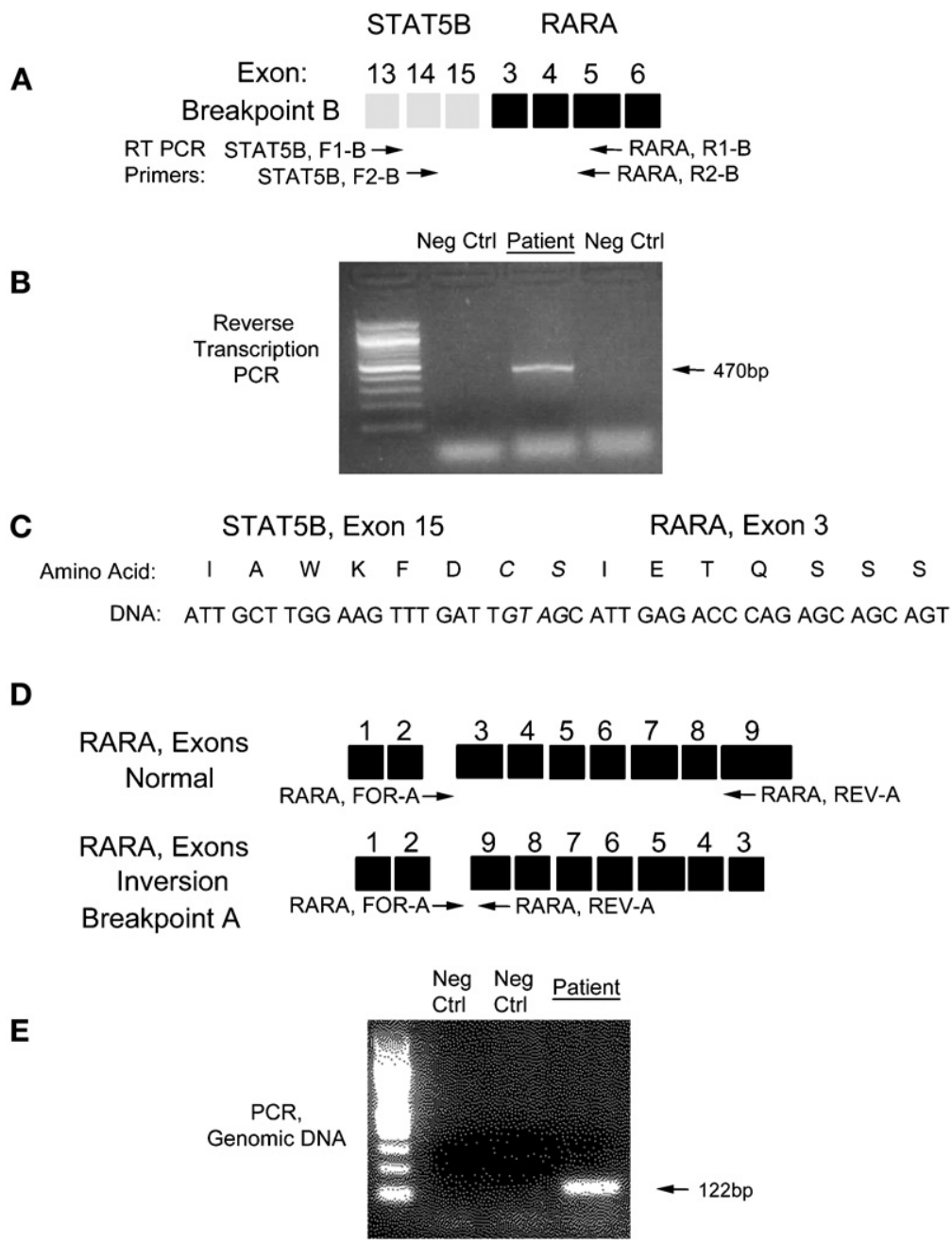

Figure 3. Inferred structure of a STAT5B-RARA and RARA-RARA inversion associated with atypical APL. (A) STAT5B-RARA fusion product. The predicted organization of the STAT5B-RARA fusion is shown with corresponding exon numbers and the positions of PCR primers used in RT-PCRs. (B) Gel showing RT-PCR product of the expected size using RNA obtained from the patient marrow but not in two negative control lanes. A 100bp molecular weight ladder is at left. (C) DNA sequence of the RT-PCR product showing an in-frame fusion of STAT5B exon 15 and RARA exon 3. The fusion transcript joint encodes two extra amino acids (italics). (D) Organization of RARA in the normal (wild-type) configuration and after inversion. Locations of primers used in PCR of genomic DNA are indicated as horizontal arrows. (E) Identification of a RARA-RARA fusion involving exon 9 and intron 2. Gel showing PCR product of expected size with patient sample DNA and not with control DNA.

$3^{\prime}$ RARA sequences. This FISH pattern has been previously reported in cases of APL with STAT5B-RARA rearrangement (Chen et al. 2012).

Given these molecular findings, the patient was given a diagnosis of atypical APL associated with a STAT5B-RARA fusion gene. Because the patient was stable and the morphologic features associated with this case were highly unusual, an additional 2-wk trial of ATRA was given, without any improvement in peripheral blood counts. The patient was then switched to standard induction chemotherapy with daunorubicin and cytarabine. Bone marrow examinations on days 15 and 45 following chemotherapy showed persistently hypercellular marrows that were unchanged morphologically from prechemotherapy marrow examinations, 
Table 3. Primers used for confirmatory PCR amplifications

\begin{tabular}{lcc}
\hline \multicolumn{1}{c}{ Name } & \multicolumn{1}{c}{ Sequence } & \multicolumn{1}{c}{ Location } \\
\hline Primer sequences for PCR, breakpoint A & \\
RARA F-A & 5' CAGTGCTGGGGGAGGTTAAA 3' & Intron 2 RARA \\
RARA R-A & 5' CGCTGAAGATGGAGATCCCG 3' & Exon 9 RARA \\
Primer sequences for PCR, breakpoint B & \\
STAT5B F1-B & 5' GCCGTGCCTGACAAAGT 3' & Exon 13 STAT5B \\
RARA R1-B & 5'TCTTCTTGTTTCGGTCGTT 3' & Exon 5 RARA \\
STAT5B F2-B & 5'ACATCTCAAGCCTCATTGGA 3' & Exon 14 STAT5B \\
RARA R2-B & 5'TGTTTCGGTCGTTTCTCAC 3' & Exon 5 RARA \\
\hline
\end{tabular}

$\mathrm{PCR}$, polymerase chain reaction.

findings that were accompanied by continued leukocytopenia and anemia. Repeat FISH analysis with the break apart RARA probe set confirmed persistence of an abnormal RARA signal in 89 of 100 cells scored.

Because of refractory disease, allogeneic matched related donor stem cell transplantation was performed following conditioning with busulfan and cytoxan. Blood counts recovered by 4-wk post-transplant. Five months post-transplant mild upper gastrointestinal symptoms prompted esophagogastroduodenoscopy, which revealed biopsy-proven inflammation consistent with mild graft-versus-host disease. The patient is currently 9-mo post-

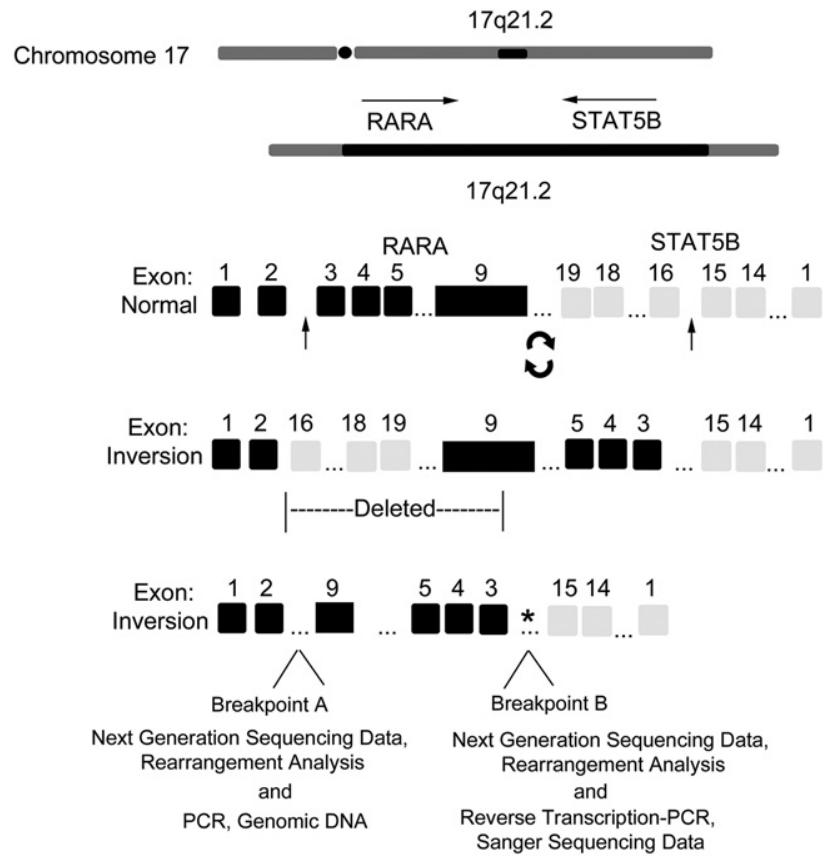

Figure 4. Inferred structure of a STAT5B-RARA inversion associated with atypical APL. STAT5B and RARA are both located at 17q21 in opposing directions of transcription. Breakpoints in STAT5B intron 15 and RARA intron 2 (arrows) followed by inversion of the intervening DNA segment led to a STAT5B-RARA fusion accompanied by deletion of part of the inverted DNA corresponding to STAT5B exon 16 and RARA exon 9. Asterisk marks sequences around breakpoint B where NGS identified an additional intronic 67-bp deletion and an intronic 42-bp inversion (not shown). 
COLD SPRING HARBOR Molecular Case Studies
STAT5B-RARA fusion and ATRA-resistance mutations

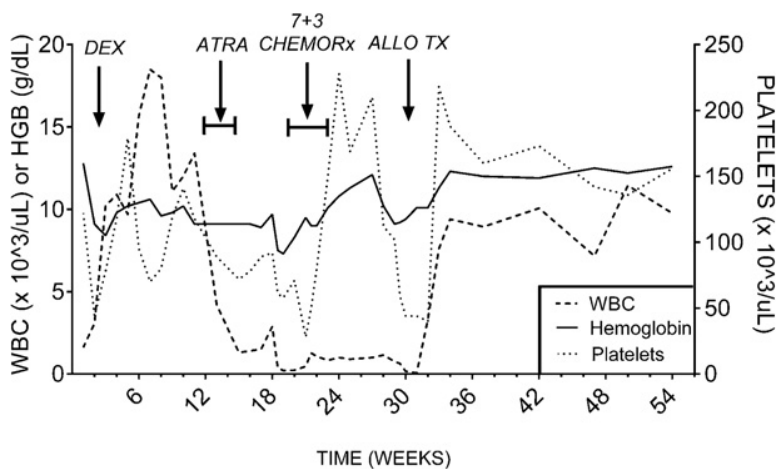

Figure 5. Case time line. Complete blood counts are shown in reference to treatment with dexamethasone (DEX), ATRA, chemotherapy $(7+3)$, and allogeneic transplantation (ALLO TX).

transplant and is in a complete hematologic remission, defined as normal peripheral complete blood counts without transfusions. Chimerism studies that assess polymorphic short tandem repeat loci have shown $96 \%-100 \%$ donor engraftment, with 100\% of leukocytes being of donor origin 9-mo post-transplant (test sensitivity, $\pm 2 \%$ ). A timeline summarizing the patient's course to date is shown in Figure 5.

\section{DISCUSSION}

This case highlights the ability of NGS to identify unexpected findings that impact diagnosis and treatment and provide insight into molecular pathogenesis. In this difficult case of a patient with a suspected variant of APL, the identification of a STAT5B-RARA gene fusion by the OncoPanel assay prompted appropriate confirmatory testing and led to definitive diagnosis and appropriate clinical management. APL associated with STAT5B-RARA is very rare (9 cases, including this one) (Arnould et al. 1999; Kusakabe et al. 2008; Swerdlow et al. 2008; Iwanaga et al. 2009; Chen et al. 2012; Strehl et al. 2013). The previously reported cases of APL with STAT5B-RARA fusion genes also showed breakpoints in STAT5B intron 15 and RARA intron 2, leading to a fusion transcript in which STAT5B exon 15 is joined in frame to RARA exon 3, as in this case. Morphologically, prior cases of APL associated with STAT5B-RARA fusion genes have had features typical of either hypergranular APL (hypergranular cytoplasm, numerous Auer rods, including multiple Auer rods within single cells) or microgranular APL (bilobed nuclei, variable cytoplasmic granularity, occasional cells with numerous Auer rods). The current case was unusual in that there was a predominance of myelocytes; hypergranular promyelocytes were not a prominent feature, and only very rare promyelocytes with single Auer rods were seen. Our experience suggests that evidence of RARA gene rearrangements should be sought in such atypical cases. At a minimum, it is important to perform FISH not only with probes designed to detect PML-RARA gene fusions (which may be cytogenetically cryptic (Welch et al. 2011)), but also with RARA break apart probes. The latter can identify RARA $3^{\prime}$ deletions, such as the one present in our case, which commonly accompany STAT5B-RARA rearrangement (Arnould et al. 1999; Kusakabe et al. 2008; Swerdlow et al. 2008; Iwanaga et al. 2009; Chen et al. 2012; Strehl et al. 2013).

STAT5B-RARA-rearranged APL tends to occur in middle age to elderly male patients presenting with anemia, thrombocytopenia, widely variable white blood cell counts, and evidence of coagulopathy (including disseminated intravascular coagulation). These cases typically showed a lack of response to ATRA and arsenic trioxide. To the best of our knowledge, this is the first case of STAT5B-RARA-rearranged APL where both breakpoints have 
been characterized. We found that one of the breakpoints in this case leads to a fusion transcript in which STAT5B exon 15 fused to RARA exon 3, as in other cases. Additionally, characterization of the other breakpoint in this case revealed that most of RARA exon 9 (encoding L409 to P462) was lost due to deletion. Since the ATRA binding domain includes RARA exon 9 and other smaller deletions in exon 9 have been associated with secondary (acquired) ATRA resistance in APL (Tomita et al. 2013), this may have contributed to the failure of this patient's disease to respond to ATRA. In addition, a single-nucleotide variant was also identified that led to a RARA R276W substitution, which has also been reported as a cause of secondary ATRA-resistance mutation in APL (Takayama et al. 2001; Tomita et al. 2013). The presence in our case of these molecular abnormalities at presentation suggests that they confer a selective advantage that is independent of ATRA treatment. Conceivably, they may render the leukemic clone more resistant to the effects of endogenous retinoids, but other possibilities also exist. For example, they may alter protein:protein interactions so as to enhance transcriptional repression or suppress ATRA-mediated degradation of STAT5B-RARA (Dong and Tweardy 2002). The former possibility is a known effect of deletions removing the RARA AF2 domain, which is encoded by the region of RARA exon 9 that is deleted in our case. It will be of interest to test these possibilities in functional assays and to determine if other cases of STAT5B-RARArearranged APL are associated with similar types of mutations.

\section{METHODS}

Targeted exome sequencing was performed using an NGS assay ("OncoPanel") that can detect somatic mutations, copy number variations, and structural variants in tumor DNA extracted from fresh, frozen or formalin-fixed paraffin-embedded samples (Wagle et al. 2012; Abo et al. 2015). The assay covers the exonic sequences of 300 cancer genes and also surveys 113 introns across 35 genes for genomic rearrangements. DNA and RNA were isolated from bone marrow using the Qiagen DNeasy and Qiamp RNA Blood minikits, respectively. DNA obtained from bone marrow was enriched for sequences of interest using a solutionphase Agilent SureSelect hybrid capture kit and then was sequenced following library preparation with an Illumina HiSeq 2500 sequencer. For the case reported here, there were a total of $5,920,923$ aligned high quality reads and a mean of 160 aligned reads across all targeted exons, with $96 \%$ of exons having $>30$ aligned reads. Sequence reads were aligned to human reference genome GRCh37 (hg19) with the Burrows-Wheeler Alignment tool (Li and Durbin 2009) with the following parameters: -q5 - 32 -k 2 -o 1 . Aligned data were sorted, duplicatemarked, and indexed with Picard tools. Base-quality score recalibration and local realignment around insertions and deletions was achieved with the Genome Analysis Toolkit (McKenna et al. 2010; DePristo et al. 2011). Structural variants were detected from aligned sequence data using BreaKmer (Abo et al. 2015). Reverse transcription-polymerase chain reactions (RT-PCRs) were carried out on RNA obtained from bone marrow that was converted to cDNA using the iScript cDNA synthesis kit (BioRad) according to the manufacturer's recommendations. PCR reactions were performed using Phusion high fidelity DNA polymerase (New England Biolabs). Primers were obtained Eurofins Genomics. Sanger sequencing of PCR products was performed by Genewiz.

\section{ADDITIONAL INFORMATION}

\section{Ethics Statement}

Human marrow and peripheral blood samples were collected with informed consent and subsequently processed and analyzed under protocols 01-206 and 11-204, which were approved by the Institutional Review Board of the Dana-Farber/Harvard Cancer Center. 
Competing Interest Statement

The authors have declared no competing interest.

\section{Referees}

Ahmet Dogen

Anonymous

Received March 3, 2015; accepted in revised form May 5 , 2015

\section{Data Deposition and Access}

BAM files containing NGS-sequencing data are available through the Sequence Read Archive at NCBI (accession number SRR2025356). RARA-RARA and STAT5B-RARA genomic sequences immediately surrounding points of chromosomal breakage and rejoining are available from GenBank (accession numbers KR855685 and KR855686). RARA point substitutions and small indels are available from ClinVar (accession numbers SCV000222915 and SCV000222916).

\section{Author Contributions}

M.J.K., R.P.A., F.C.K., N.I.L., and J.C.A. contributed to analysis and interpretation of the next-generation sequencing data. R.D.B. designed, performed, and/or interpreted RTPCR and Sanger sequencing analyses. P.D.C. interpreted fluorescence in situ hybridization assays and cytogenetic findings. O.P. and E.A.M. interpreted flow cytometric findings and contributed micrographs. D.J.D. contributed and reviewed clinical features of the case. All authors contributed to the writing of the manuscript.

\section{Funding}

J.C.A. is supported by a grant from the National Institutes of Health (P01CA119070).

\section{REFERENCES}

Abo RP, Ducar M, Garcia EP, Thorner AR, Rojas-Rudilla V, Lin L, Sholl LM, Hahn WC, Meyerson M, Lindeman $\mathrm{NI}$, et al. 2015. BreaKmer: detection of structural variation in targeted massively parallel sequencing data using kmers. Nucleic Acids Res 43: e19.

Arnould C, Philippe C, Bourdon V, Grégoire MJ, Berger R, Jonveaux P. 1999. The signal transducer and activator of transcription STAT5b gene is a new partner of retinoic acid receptor $\alpha$ in acute promyelocytic-like leukaemia. Hum Mol Genet 8: 1741-1749.

Chen H, Pan J, Yao L, Wu L, Zhu J, Wang W, Liu C, Han Q, Du G, Cen J, et al. 2012. Acute promyelocytic leukemia with a STAT5b-RARa fusion transcript defined by array-CGH, FISH, and RT-PCR. Cancer Genet 205: 327-331.

DePristo M, Banks E, Poplin R, Garimella K, Maguire J, Hartl C, Philippakis A, del Angel G, Rivas MA, Hanna M, et al. 2011. A framework for variation discovery and genotyping using next-generation DNA sequencing data. Nat Genet 43: 491-498.

Dong S, Tweardy DJ. 2002. Interactions of STAT5b-RARa, a novel acute promyelocytic leukemia fusion protein, with retinoic acid receptor and STAT3 signaling pathways. Blood 99: 2637-2646.

Iwanaga E, Nakamura M, Nanri T, Kawakita T, Horikawa K, Mitsuya H, Asou N. 2009. Acute promyelocytic leukemia harboring a STAT5B-RARA fusion gene and a G596V missense mutation in the STAT5B SH2 domain of the STAT5B-RARA. Eur J Haematol 83: 499-501.

Kusakabe M, Suzukawa K, Nanmoku T, Obara N, Okoshi Y, Mukai HY, Hasegawa Y, Kojima H, Kawakami Y, Ninomiya $\mathrm{H}$, et al. 2008. Detection of the STAT5B-RARA fusion transcript in acute promyelocytic leukemia with the normal chromosome 17 on G-banding. Eur J Haematol 80: 444-447.

$\mathrm{Li} \mathrm{H}$, Durbin R. 2009. Fast and accurate short read alignment with Burrows-Wheeler transform. Bioinformatics 25: 1754-1760.

McKenna A, Hanna M, Banks E, Sivachenko A, Cibulskis K, Kernytsky A, Garimella K, Altshuler D, Gabriel S, Daly M, et al. 2010. The Genome Analysis Toolkit: a MapReduce framework for analyzing next-generation DNA sequencing data. Genome Res 20: 1297-1303.

StrehI S, Konig M, Boztug H, Cooper BW, Suzukawa K, Zhang SJ, Chen HY, Attarbaschi A, Dworzak MN. 2013. All-trans retinoic acid and arsenic trioxide resistance of acute promyelocytic leukemia with the variant STAT5B-RARA fusion gene. Leukemia 27: 1606-1610.

Swerdlow SH, International Agency for Research on Cancer, \& World Health Organization. 2008. WHO classification of tumours of haematopoietic and lymphoid tissues, 4th ed. International Agency for Research on Cancer, Lyon, France. 
Takayama N, Kizaki M, Hida T, Kinjo K, Ikeda Y. 2001. Novel mutation in the PML/RARa chimeric gene exhibits dramatically decreased ligand-binding activity and confers acquired resistance to retinoic acid in acute promyelocytic leukemia. Exp Hematol 29: 864-872.

Tomita A, Kiyoi H, Naoe T. 2013. Mechanisms of action and resistance to all-trans retinoic acid (ATRA) and arsenic trioxide (As2O 3) in acute promyelocytic leukemia. Int J Hematol 97: 717-725.

Wagle N, Berger MF, Davis MJ, Blumenstiel B, Defelice M, Pochanard P, Ducar M, Van Hummelen P, Macconaill LE, Hahn WC, et al. 2012. High-throughput detection of actionable genomic alterations in clinical tumor samples by targeted, massively parallel sequencing. Cancer Discov 2: 82-93.

Welch JS, Westervelt P, Ding L, Larson DE, Klco JM, Kulkarni S, Wallis J, Chen K, Payton JE, Fulton RS, et al. 2011. Use of whole-genome sequencing to diagnose a cryptic fusion oncogene. JAMA 305: 1577-1584. 


\section{COLD SPRING HARBOR Molecular Case Studies}

\section{Myeloid neoplasm demonstrating a STAT5B-RARA rearrangement and genetic alterations associated with all- trans retinoic acid resistance identified by a custom next-generation sequencing assay}

Michael J. Kluk, Ryan P. Abo, Ronald D. Brown, et al.

Cold Spring Harb Mol Case Stud 2015, 1: a000307

Access the most recent version at doi: $10.1101 /$ mcs.a000307

References This article cites 14 articles, 3 of which can be accessed free at: http://molecularcasestudies.cshlp.org/content/1/1/a000307.full.html\#ref-list-1

License This article is distributed under the terms of the Creative Commons

Attribution-NonCommercial License, which permits reuse and redistribution, except for commercial purposes, provided that the original author and source are credited.

Email Alerting Receive free email alerts when new articles cite this article - sign up in the box at the Service top right corner of the article or click here. 